\author{
Accelerator $:$ Department \\ BROOKHAVEN NATIONAL LAABORATORY \\ Associated Universities, Inc: \\ Uptọ, L.I., N.Y:
}

AGS DIVISION TECHNICAL NOTE:

No. 48
M. Fruitman and E.B. Forsyth
March 28; 1968

Until now the new fast kicker: design has been referred to as the.

$80 \mathrm{kV}$ kicker due largely to the E.B. Forsyth Technical Note 非3 of JuIy 25 ; $1966^{\circ}$ which set the maximum limit at $80 \mathrm{kV}$. This was done at the time because the highest voltage rated tube available was the CXI168 at $80 \mathrm{kV}$ and because the required deflecting force was anticipated. to be 132. kilogauss-cm.

The design described here deals with an anticipated deflecting. force of 200 kilogauss $\mathrm{cm}$ minimum and thus requires the CX1171 thyratron $\cdots$ which has been tentatively rated:at $120 \mathrm{kV}$. Furthermore, the former consideration of two pulses per AGS cycle has given way to three pulses per post conversion AGS cycle. This is a three-fold increase to three pulses-per second for: partial extractions. The previous specification. of "any number of bunches"' has been limited, due to practical considera-. tions, to any number up to four per kicker pulse. The previously proposed "tailbiter"! circuit will still be used to accomplish this variable bunch feature. Ful1 extraction will still be possible through manual switching: of the PFN and tailbiter. A layout is now planned such that the magnets, feedthroughs, load resistors and a polarity switching device are in the AGS tunne 1.:. A11 other components will be located in 
a "cool"."area outside.

\section{The New Design}

The new design"starts from the following premises:

1) The minimum required deflecting force is $200 \mathrm{ki}$ logaus $-\mathrm{cm}$ for the post conversion beam......

2)...The maximum tolerable risetime is 200 nanoseconds due to the time between bunches:

3). The maximum usable magnet length in a 10 ft straight section is 80 inches or approximately: $200 \mathrm{~cm}$.

4). The maximum usable voltage at this time is $120 \mathrm{kV}$. which is the, maximum forward voltage rating on the CX1171:

5) The maximum usable current at this time is 8000 amperes which is optimistically based on the present operation of the CX1168 at 6500 amperes.

Three further conditions are added, based on the experience with the present:operating fast.kicker: :

1). The present magnet cross section of $2-5 / 8$ inches $(6.65 \% \mathrm{~cm})$ high by 6 inches $(15.25 \mathrm{~cm})$ wide is satisfactory and using these dimensions in a1l computations, neglecting conductor and insulation.... thickness, has proven satisfactory within our accuracy of design:

2) The stray inductance in the circuit inc luding the tube, Ioad ... resistors and connections can be expected to approach 875 "nanohenries.

3) The effective magnet length for purposes of computation is assumed to be 1.5 times the height longer than the actual magnet length. Thus, each magnet used is effectively $10 \mathrm{~cm}$ longer, based on our magnet height: 
Two safety factors were used in all calculations:

1) 175 nanoseconds was used to assure a risetime of less than 200 "nanosec onds.

2) A worst case design goal of 250 kilogauss $-\mathrm{cm}$ was chosen to yield an expected nominal deflection force of 200 kilogauss-cm.

The specifications and Iimitations noted above leave only a few. variables, a11 interrelated by the accepted design formulas. Computations were made for each of the cases where the total magnet was composed of one through five equal magnet sections and the results were tabulated. of these possibilities only the case with four magnets fell within the limits of current, voltage and length previously specified: This case, with each magnet driven by a separate switch tube, yields an optimum:of 7.58 ohms load impedance with a magnet length of $128 \mathrm{~cm}$ and a current of 7900 amperes at i $120 \mathrm{kV}$..

Additional calculations were made for a similar circuit, but using 7 "ohms which. would be available based on the nominal impedance of 14 "ohms. for $100 \mathrm{P} 2 / 2$ cable. Within the 8000 ampere maximum current this forces a compromise of either the $250 \mathrm{kilogauss-cm}$ deflecting force or the 17.5 nanosecond risetime: The following summary of proposed characteristics Is the result of that compromise and is the design goal for the prototype system which wi11 consist of one quarter of the"final system. 


$$
-4-
$$

Summary of Proposed Specifications

Nominal Deflecting. Force, Maximum: 200 kilogauss-cm

Rise and Fall Time ( $5 \%$ points): 190 nanosec:

Flät Top Length:

Infinitely variable; 0-660 nanosec. or 2.7 microsec. (manually. switched).

Maximum Number of Fast Kicker. Pulses Per AGS Cycle: : Partial extraction - 3 Ful1 extraction - 1

Minimum Time Between Pulses: Partial extraction - 100 millisec.

$$
\text { Ful1 extraction-... 500 millisec: }
$$

Magnet : aperture:

$6.65 \mathrm{~cm}$ high $\mathrm{x} \cdot 15.25 \mathrm{~cm} \cdot \mathrm{wide}$

Magnet length, total:...

$126 \mathrm{cms}$

Polarity:

reversible in 8 hours

Field stability and ripple, max.: $1 \%$.

No. of imagnet sections: $\quad 4$

No. of switch tubes: 4

No: of tailbiter tubes: 4

Operating volts at nominal deflecting force: $100 \mathrm{kV}$

Deflecting force control, partial extraction: first, second and third pulse per AGS cycle independently ad justable.

Distr:

Accelerator Department Staff: : 


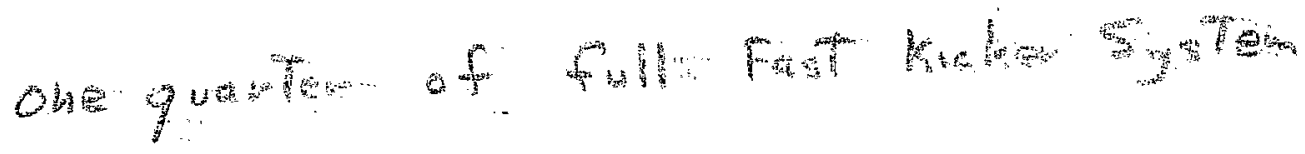

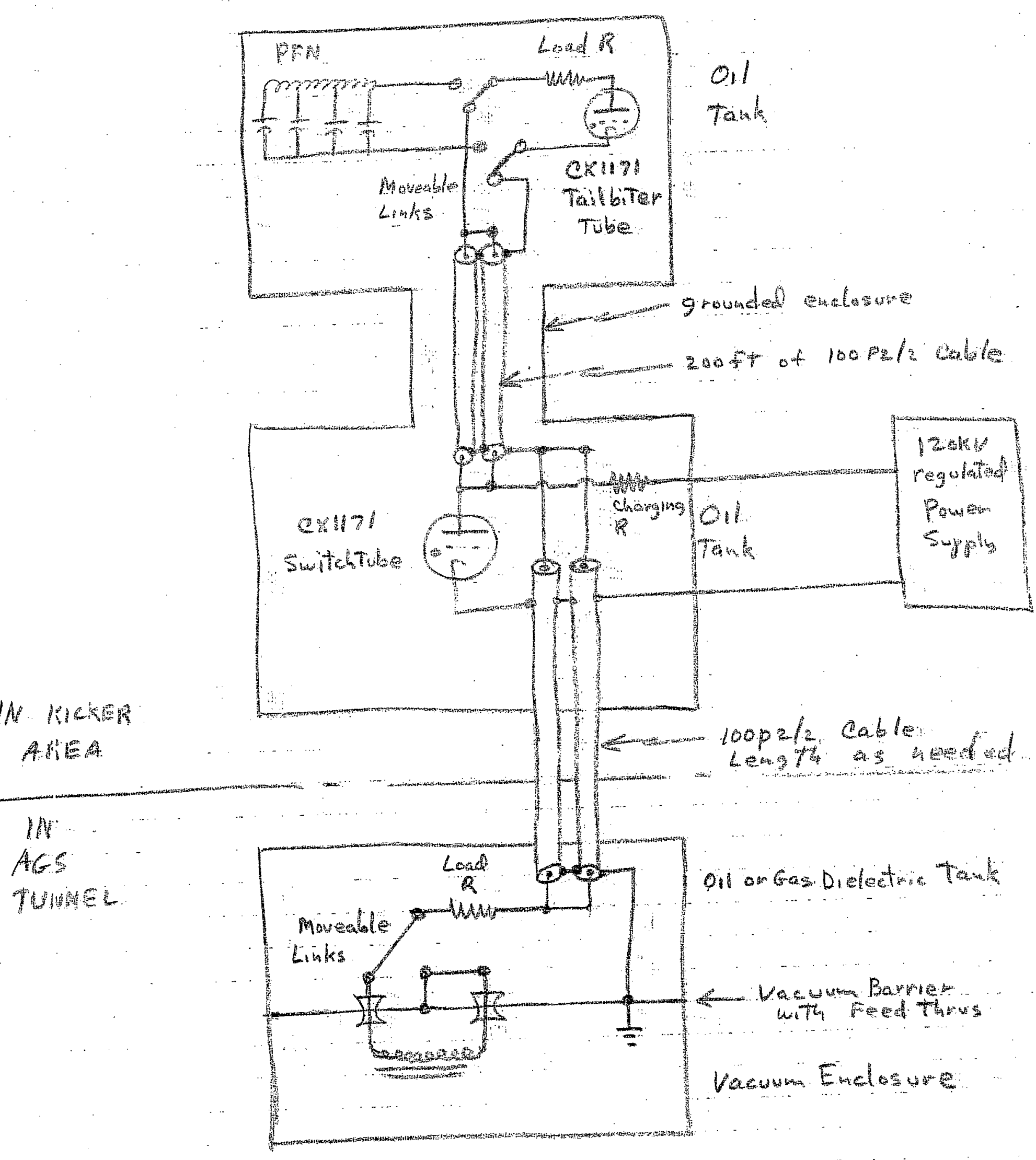

